\title{
Lung Segmentation Using Deep Learning
}

\author{
S. Vinitha Sri ${ }^{1} \&$ S.P. Kavya ${ }^{2}$ \\ ${ }^{1,2}$ Department of Computer Science and Engineering, Sri Shakthi Institute of Engineering and Technology, Coimbatore, India. \\ DOI: http://doi.org/10.38177/ajast.2021.5202
}

Copyright: @2021 S.Vinitha Sri \& S.P.Kavya. This is an open access article distributed under the terms of the Creative Commons Attribution License, which permits unrestricted use, distribution, and reproduction in any medium, provided the original author and source are credited.

Segmentation is useful in different applications mainly it plays vital role in medical analysis. For image analysis of lungs, segmentation is an important step, thus it plays a major role in efficient image processing. Lung Segmentation helps to improve the early finding and treatments of lung infections. The performance of analysis and diagnosis of lung issues can be improved and it lessen the work of radiologists.

The U-Net architecture was introduced mainly for Image segmentation in Biomedical. This architectures are mainly consists of both encoders and decoders which is most important for image segmentation. The neural networks shows better performance when the network is initializes with weights which are pre-trained on huge dataset like ImageNet. The aim of this paper is to develop a system that performs automatic lung segmentation procedure to determine the boundaries of lobes from surrounding thoracic tissue using Deep Learning and to compare the U-Net and U-Net using VGG11 encoder pre-trained on ImageNet with the existing systems. The proposed system using the U-Net architecture and U-Net with VGG-11 encoder pre-trained on ImageNet achieved the accuracy of $97.63 \%$ and $98.28 \%$ respectively.

Keywords: Fully convolutional networks (FCN), Convolutional neural network (CNN), Image segmentation, U-Net, Visual Geometry Group-11 (VGG-11), Chest X-ray (CXR), Refined linear unit (ReLU), Deep learning (DL), Machine learning (ML), Artificial intelligence (AI).

\section{Introduction}

The objective of segmentation is to rearrange and additionally change the view of an image into something that is more significant and simpler to analyse. Segmentation is used to find boundaries and objects in images. It is considered the most fundamental clinical imaging as it extracts region of interest through automatic process. Semantic image segmentation classifies every pixel of an image with a relating class.

The actual yield is a high-resolution image in which every pixel is characterized to a specific class. Along these lines it is a pixel level image categorization. More patients tend to have an x-ray check-up at hospitals, which adds more workload to radiologists so here the process of lung segmentation is highly beneficial. Lung Segmentation helps to improve the early finding and treatments of lung infections. $\mathrm{CNN}$ is trained in end to end way for the segmentation of the input images in this method.

Also this helps in improving the accuracy in segmentation over some common datasets. Further improved verson of this method is called as U-Net neural network and a further improvisation has been made by replacing encoder of UNet with VGG11 encoder pretrained on Imagenet. This paper is structured as follows: The introduction is given in section 1. Section 2 presents literature survey. Algorithms were explained in section 3. In section 4, the proprosed methodology and the flow diagram is explained and the result is shown in section 5. Finally the result is given in section 6.

\section{Literature Survey}

[1] Hieu Thrung Huyn and Vo Nguyen Nat Ana proposes a deep learning method for segmenting the lung on large size chest X-ray image. This paper proposes a network architecture for lung segmentation using CNNs and traditional neural networks. The boundaries of lung region are not smooth as the other methods because the 


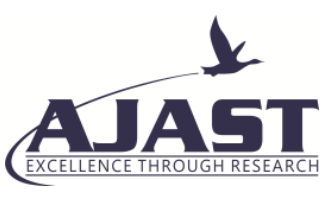

Asian Journal of Applied Science and Technology (AJAST)

Volume 5, Issue 2, Pages 10-19, April-June 2021

network classified each pixel. [3] Mingje Xu,Souliang Qi, Yong Yueh, Yueyangh Teng, Lisheng Xuh ,Yhudong Yao ,Wei Qian 2019(Springer Paper). This method proposes a convolutional neural network (CNN) model to segment lung parenchyma. It does not work well with clusters of different size and density. [5] Cing-Sheng Chang, Jin-Fah Lin, Ming - Cing Lee, Christop Palm ,2020(Springer Paper) uses the architecture of DeepLabv3+ which is well known as state-of-art in the PASCAL VOC 2012 dataset. This work uses NLH Chest X-ray dataset and applies a neural network architecture for automatic lung region segmentation. The bilinear up sampling method may be not good enough and to gain more information, maybe more features from the inner layers should be concatenated with the low-level feature.

\section{Algorithms}

\subsection{Deep Learning}

Deep learning is a function that copies the human brains working in handling information and recognizing patterns which benefits in making decisions. It comes within the category of ML in AI. A large dataset and significant processing power is required by Deep Learning.

\subsection{Fully Convolutional Networks}

In order to convert image pixels into pixel classifications a fully convolution network makes use of the convolution neural network. The width and height of the intermediate layer is converted by the FCN to the input image's actual size as shown in Fig 1. FCN uses filters for learning. Given a pixel, the output will be a classified prediction of the corresponding pixel.

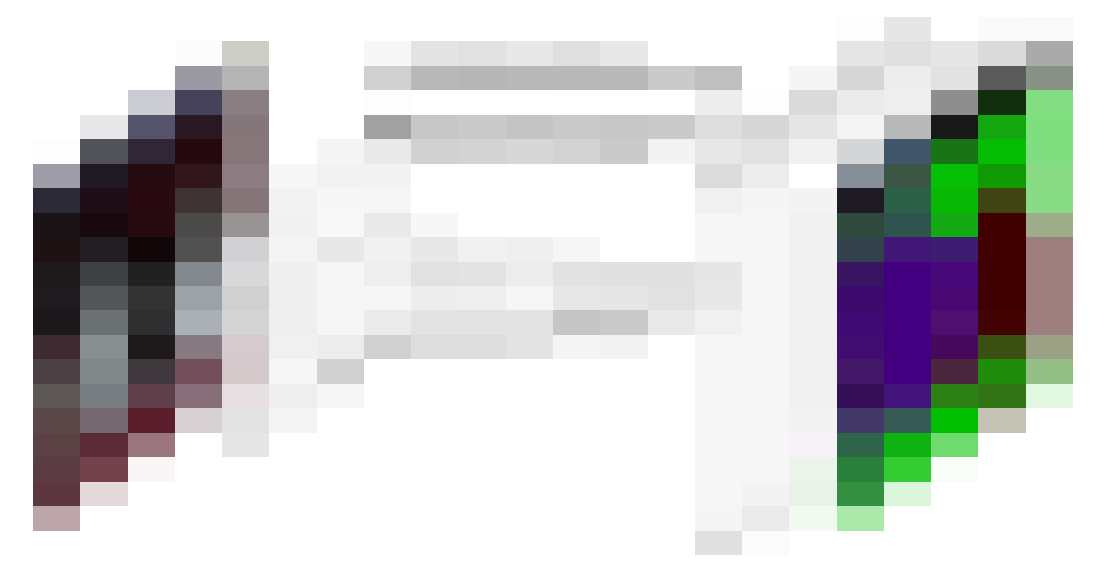

Fig.1. Fully Convolutional Network

\section{Proposed Methodology}

\subsection{U-Net}

The U-Net architecture is the best so far introduced for Biomedical Image segmentation. The architecture is composed of two main parts that are encoder and decoder. The encoder (compressive part) is used to extract the features in the image. The decoder (expansive part) uses transposed convolution to bring back the image to the actual size and promote segmentation. It is also called as "Fully Convolutional Neural Network" with no fully connected layers. The encoder consists of the repeated application of two $3 \times 3$ unpadded convolutions, each 


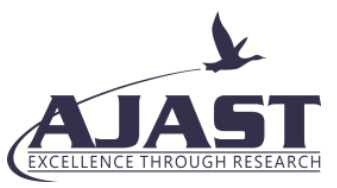

Asian Journal of Applied Science and Technology (AJAST)

Volume 5, Issue 2, Pages 10-19, April-June 2021

followed by a rectified linear unit (ReLU) and for down sampling a $2 \times 2$ max pooling operation with stride 2 . The number of feature channels are doubled at each down sampling. The encoder captures the context in the image. The decoder is the symmetric to that of the encoder and it is used to empower precise localization using transposed convolutions. This architecture is made up of 23 convolutional layers. The U-Net architecture is shown in Fig 2.

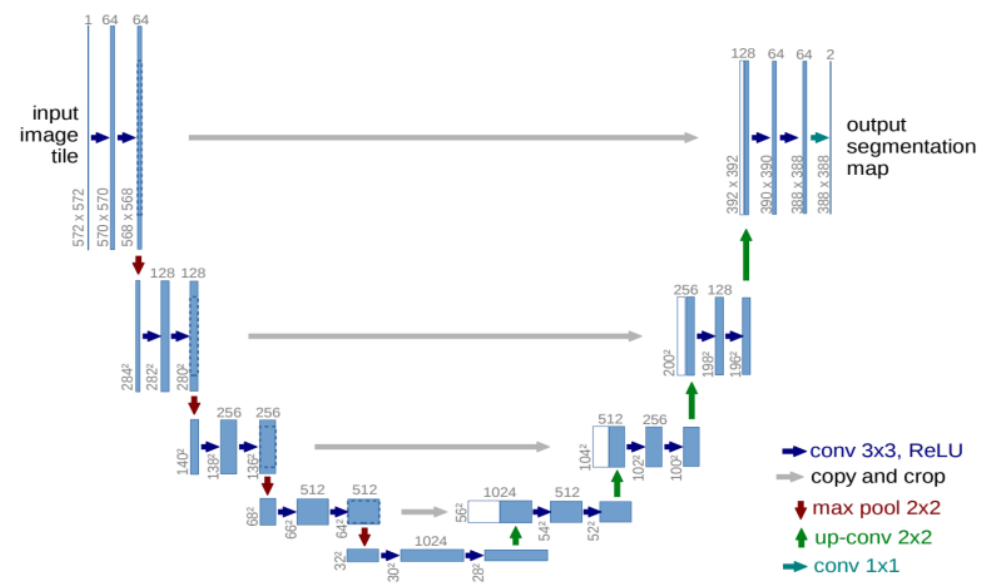

Fig.2. U-Net Architecture

\subsection{VGG-11}

\subsubsection{VGG-11 Architecture}

VGG means Visual Geometry Group. It is a model which is pretrained on Imagenet, over a large dataset and contains the weights which represents the features of whichever dataset it was trained on. Using a pre-trained model saves time. This model takes input as $224 \times 224$ pixel image in RGB format. It uses a 3x3 convolution layer followed by $2 \times 2$ maxpooling which returns the maximum value from the pixel set.The activation function used here is the ReLU. The Softmax activation function is used for the conversion of numbers into respective probabilities. The VGG-11 architecture is shown in Fig. 3.

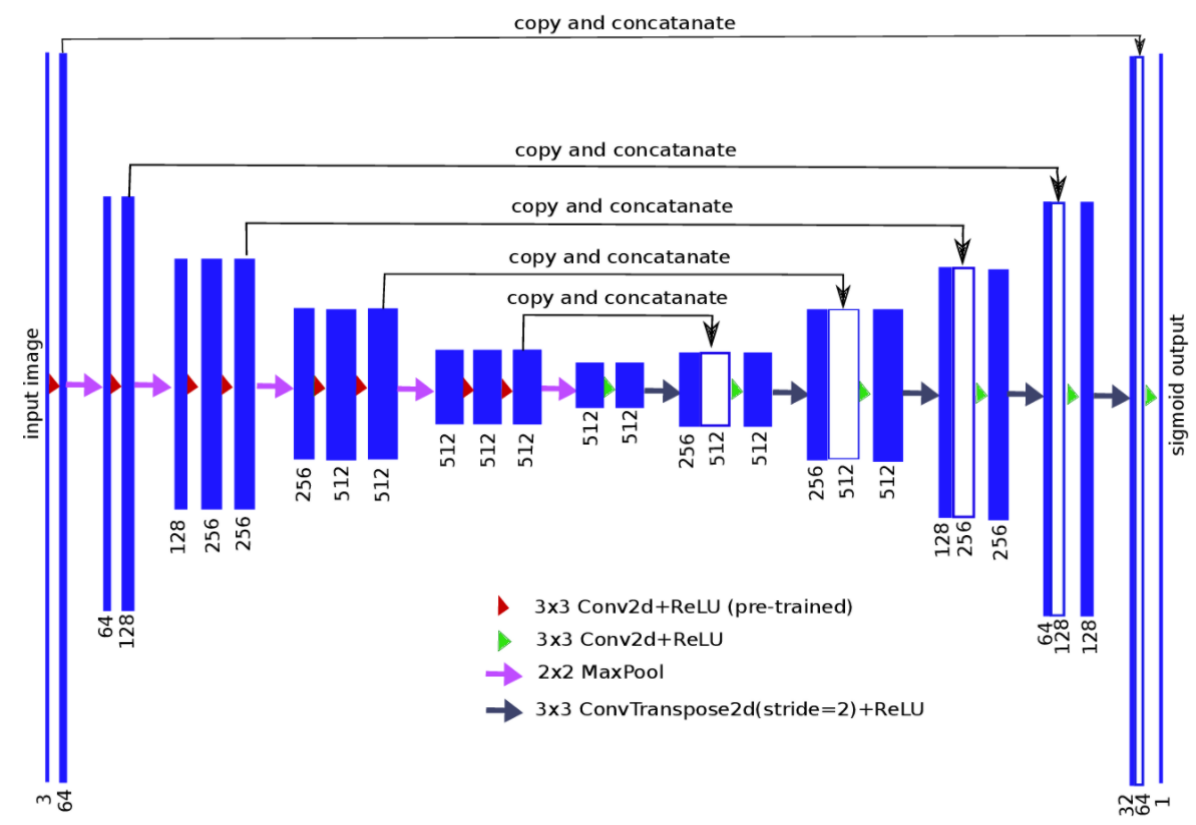

Fig.3. VGG-11 architecture 


\subsubsection{The VGG-11 layers}

The VGG-11consists of 11 layers which is shown in Fig. 4.
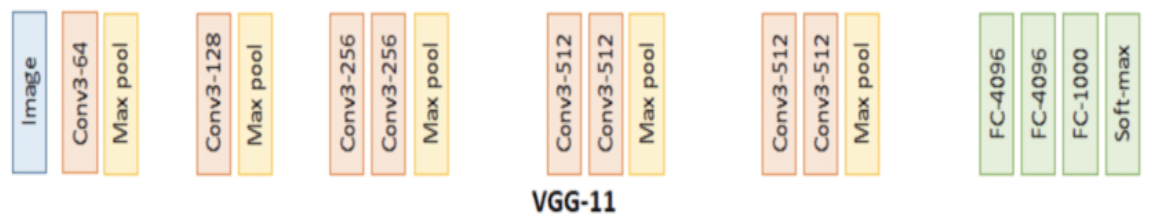

Fig.4. VGG-11 layers

\subsection{Flow Diagram}

The Shenzen Lung Segmentation dataset and their corresponding masks are loaded from disk. The input image is preprocessed and given as an input to build the Lung Segmentation model. The Lung Segmentation model is built and trained on the dataset using Keras and Tensorflow. The model is tested to obtain the accuracy of the Lung Segmentation model. If the obtained accuracy is greater than or equal to the estimated accuracy, the model is saved. Otherwise, the model is retrained by varying the parameters. The accuracy and loss curves are plotted using graphs. The flow diagram for building the Lung Segmentation model is shown in fig 5 .

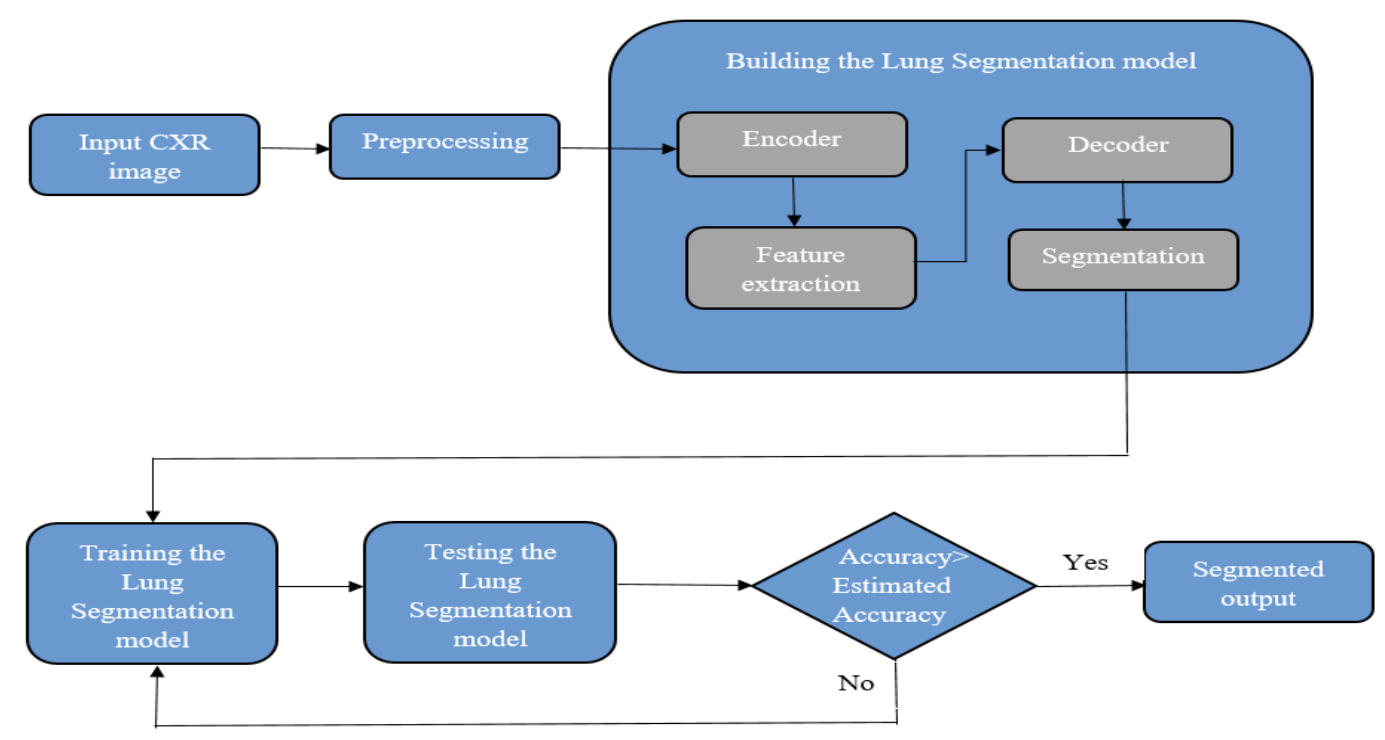

Fig.5. Flow diagram for building a Lung Segmentation model

\subsubsection{Dataset}

The Chest X-Ray dataset has been collected from the kaggle repository. The dataset consists of 2128 images. It consists of 1064 Chest X-Ray images and 1064 mask images as shown in Fig 6 and Fig 7.

\subsubsection{Training the dataset using $U$-Net and VGG11 pretrained on ImageNet}

The dataset is trained, and a model is developed using the U-Net - Deep learning architecture to determine the boundaries of lobes from surrounding thoracic tissue. The dataset is trained, and a model is developed using U-Net with VGG11 encoder pre trained on ImageNet- Deep learning architecture to determine the boundaries of lobes from surrounding thoracic tissue. 
AJAST

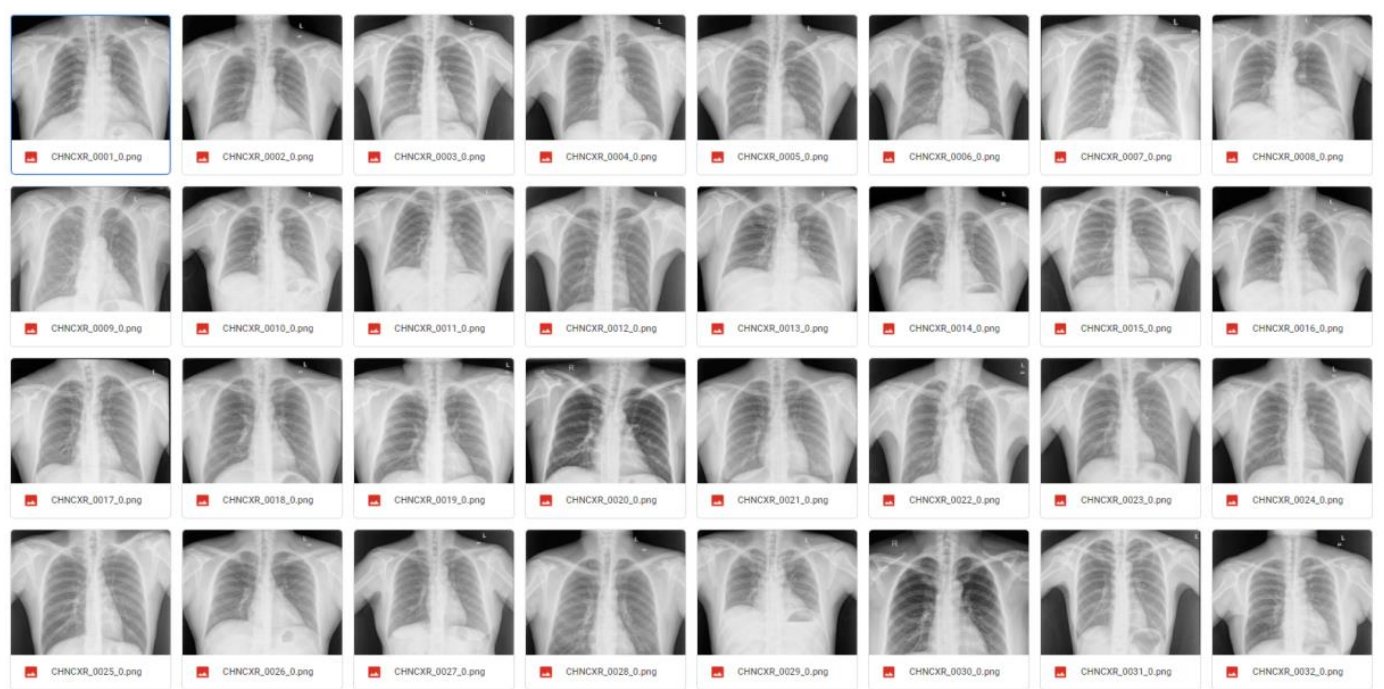

Fig.6. Chest X-Ray

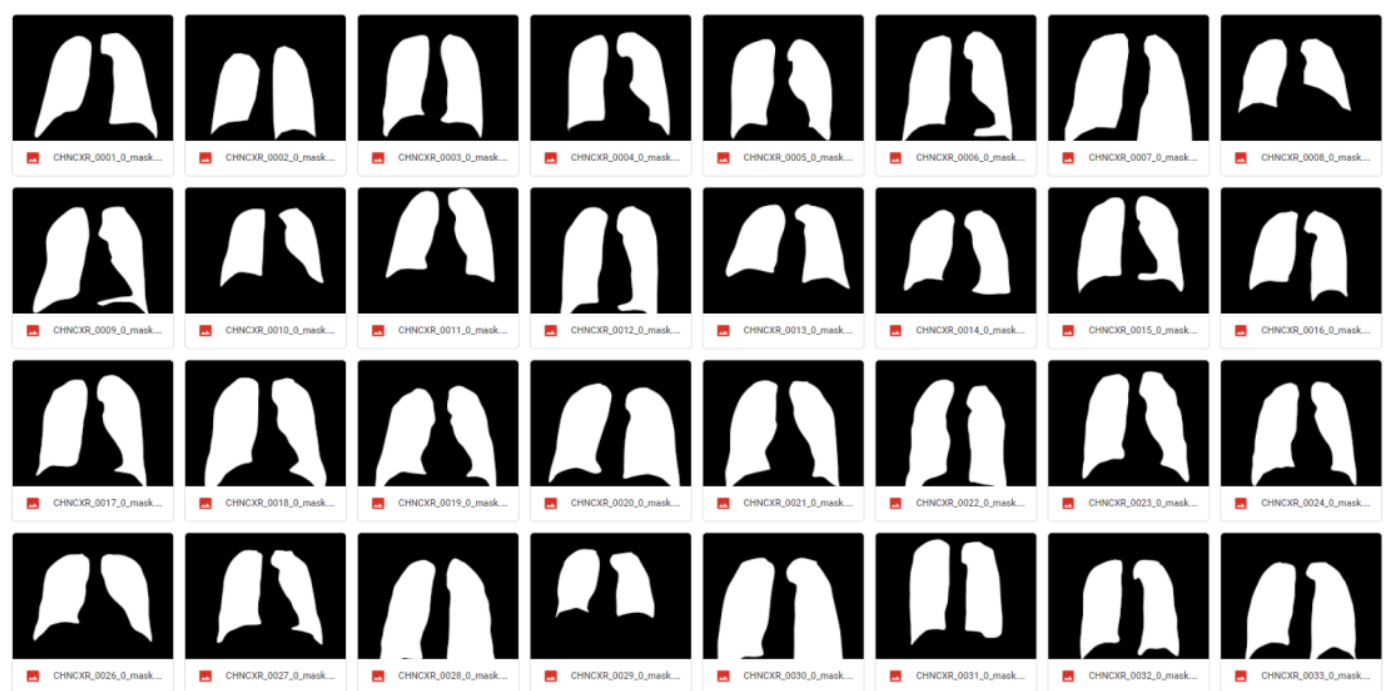

Fig.7. Masks

\subsubsection{Data Preprocessing}

\section{U-Net}

In data preprocessing, the dataset images resized to $512 \times 512$ and the preprocessing output is shown in Fig. 8
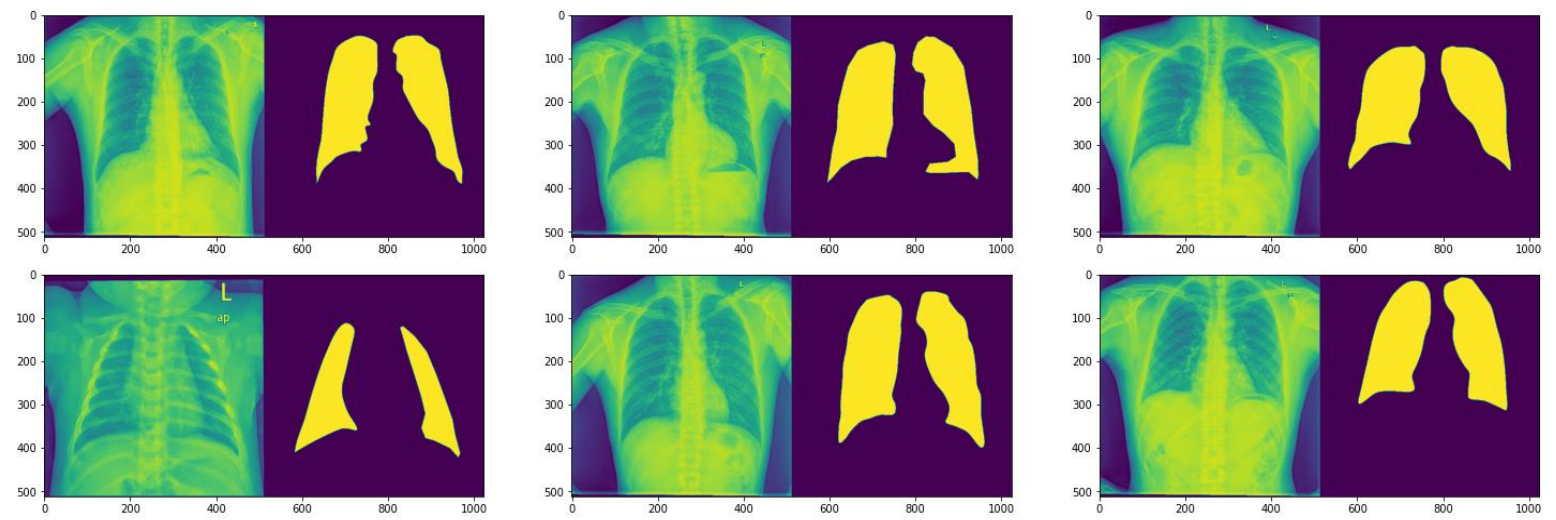

Fig.8. Pre-processing output 


\section{VGG11 pre-trained on ImageNet}

In data preprocessing, the dataset images are resized to $512 \times 512$ and then padded and cropped and the preprocessing output is shown in Fig 9.
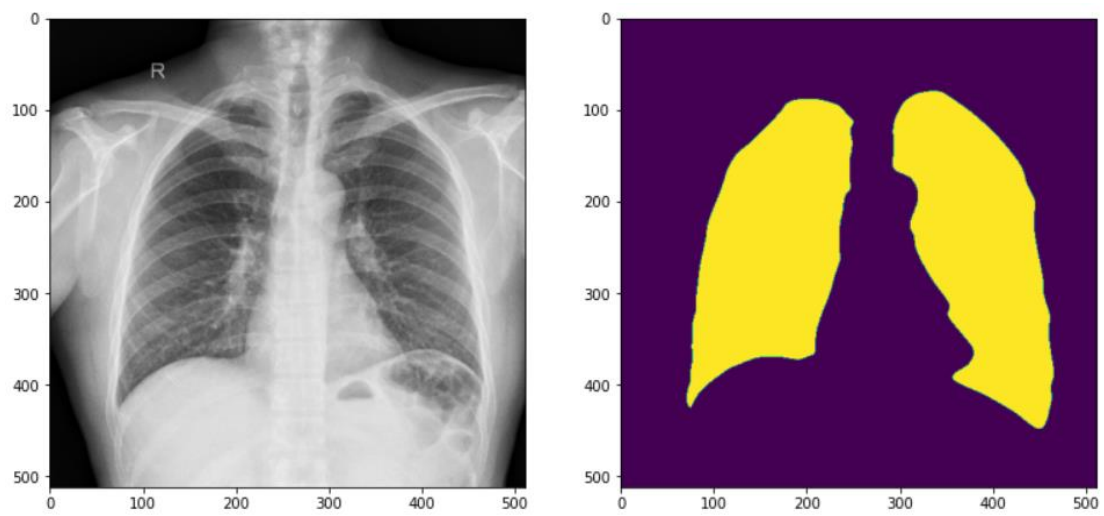

Fig.9. Preprocessing output

\subsubsection{Lung Segmenter Model parameters}

\section{U-Net}

Initial Learning Rate: The rate of learning issues can be controlled by the learning rate parameter. If it is minimum, the loss will be also minimum. For the Lung Segmenter model, the initial learning rate value is set to 0. 0002. The learning rate is shown in Fig.10.

Epochs: The number of iterations the learning algorithm go through is the Epochs. For the Lung Segmenter model, the epoch is set to 10 .

Batch Size: The batch size characterizes the quantity of tests to work through prior to refreshing the internal model boundaries. For the Lung Segmenter model, batch value is set to 32. The Epochs/Batch Size are shown in Fig. 11.

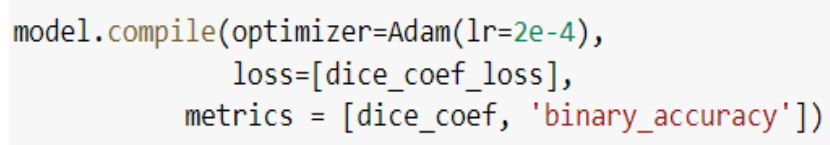

Fig.10. Learning Rate

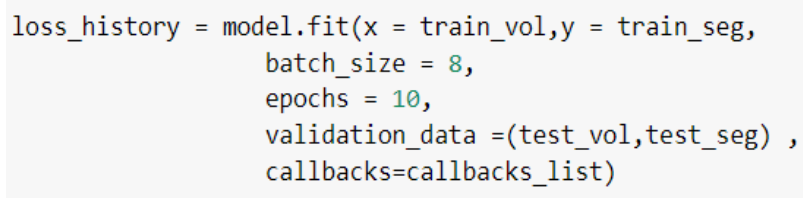

Fig.11. Epochs and Batch Size

\section{VGG11 pretrained on ImageNet}

Initial Learning Rate: The rate of learning issues can be controlled by the learning rate parameter. If it is minimum, the loss will be also minimum. For the Lung Segmenter model, the initial learning rate value is set to 0. 0002. The learning rate is shown in Fig. 12. 


\section{AJAST}

Asian Journal of Applied Science and Technology (AJAST)

Volume 5, Issue 2, Pages 10-19, April-June 2021

Epochs: The number of iterations the learning algorithm go through is the Epochs. For the Lung Segmenter model, the epoch is set to 8 .

Batch Size: The batch size characterizes the quantity of tests to work through prior to refreshing the internal model boundaries. For the Lung Segmenter model, the batch value is set to 4. The Epochs and Batch Size are shown in Fig. 13.

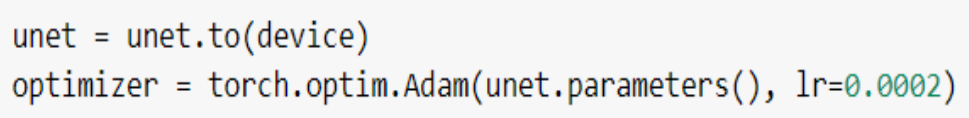

Fig.12. Learning Rate

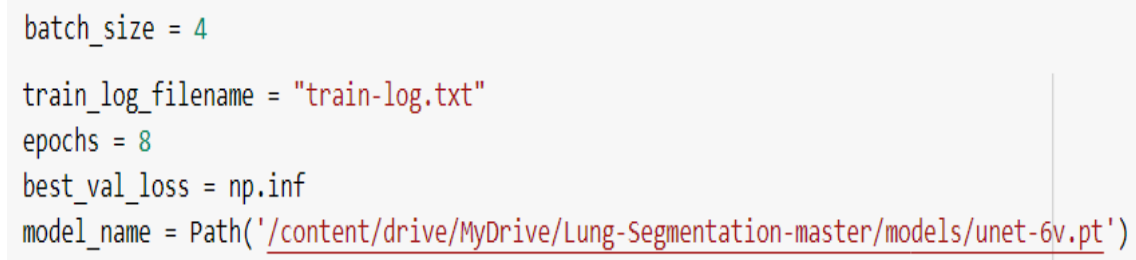

Fig.13. Epochs and Batch Size

\section{Results}

The loss and the accuracy plot have been plotted. Lung Segmenter's training accuracy and loss curves demonstrate high accuracy and little signs of overfitting on the data as shown in Fig.14 and Fig. 15. The model becomes ready to perform Lung Segmentation. The accuracy of the model obtained for U-Net is $97.63 \%$ on the dataset and for U-Net with VGG-11 encoder pre-trained with ImageNet is $98.28 \%$.
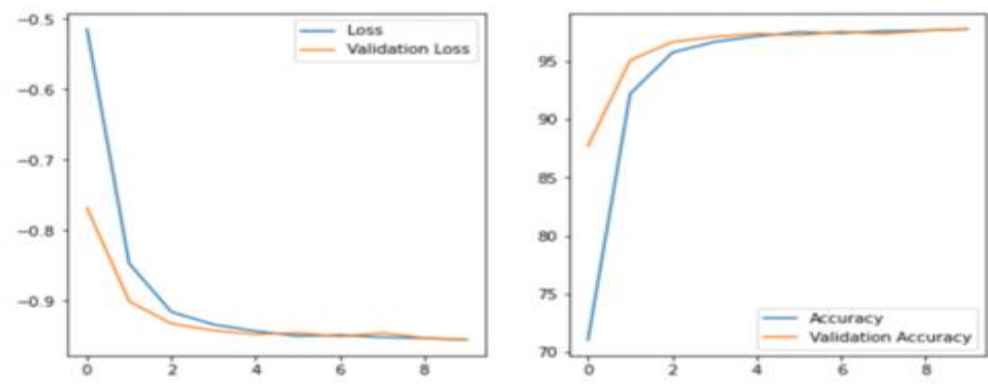

Fig.14. Loss and accuracy plot of UNet

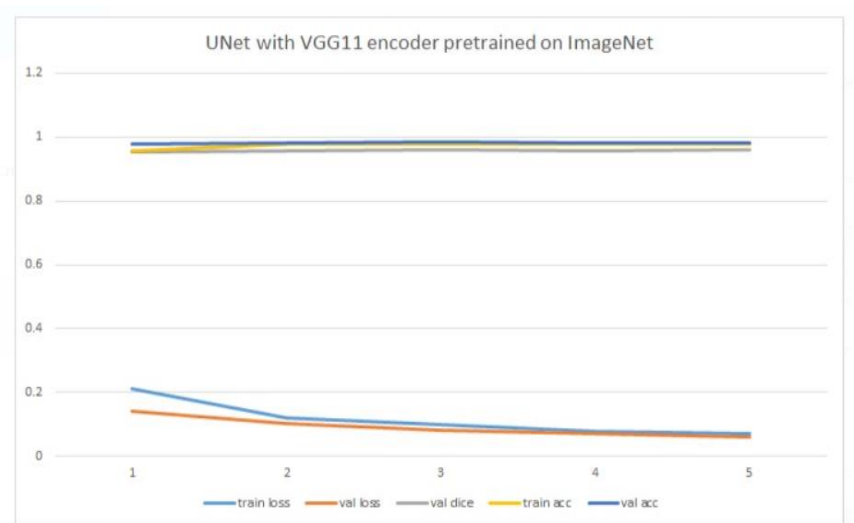

Fig.15. Loss and Accuracy of U-Net with VGG-11 encoder pre-trained with ImageNet 


\section{AJAST}

Asian Journal of Applied Science and Technology (AJAST)

Volume 5, Issue 2, Pages 10-19, April-June 2021

\subsection{Comparison Between Existing and Proposed System}

The comparison between the architectures such as GCN, VGG Net, HDC/DCUC, U-Net and U-Net with VGG-11 encoder pre-trained with ImageNet based on the learning rate, epochs, dice coefficient and its accuracies are shown as the following Table and Figures:

Table 1: The table compared for existing and proposed system

\begin{tabular}{|c|l|l|l|l|l|}
\hline Architecture used & $\begin{array}{c}\text { Learning } \\
\text { rate }\end{array}$ & Epochs & \multicolumn{1}{|c|}{$\begin{array}{c}\text { Dice } \\
\text { coefficient }\end{array}$} & $\begin{array}{c}\text { Training } \\
\text { accuracy } \\
\text { (\%) }\end{array}$ & $\begin{array}{c}\text { Testing } \\
\text { accuracy } \\
\text { (\%) }\end{array}$ \\
\hline \multicolumn{6}{|c|}{ Existing system } \\
\hline GCN & $4 \mathrm{e}-5$ & 30 & 0.9070 & 93.15 & 93.26 \\
\hline VGG Net & $1 \mathrm{e}-4$ & 75 & 0.9523 & 96.26 & 96.19 \\
\hline HDC/DCUC & $1 \mathrm{e}-5$ & 100 & 0.8501 & 91.42 & 91.23 \\
\hline \multicolumn{6}{|c|}{ Proposed system } \\
\hline $\begin{array}{c}\text { UNet with VGG-11 encoder } \\
\text { pretrained on ImageNet }\end{array}$ & $2 \mathrm{e}-4$ & 5 & 0.9617 & 97.94 & 98.28 \\
\hline
\end{tabular}
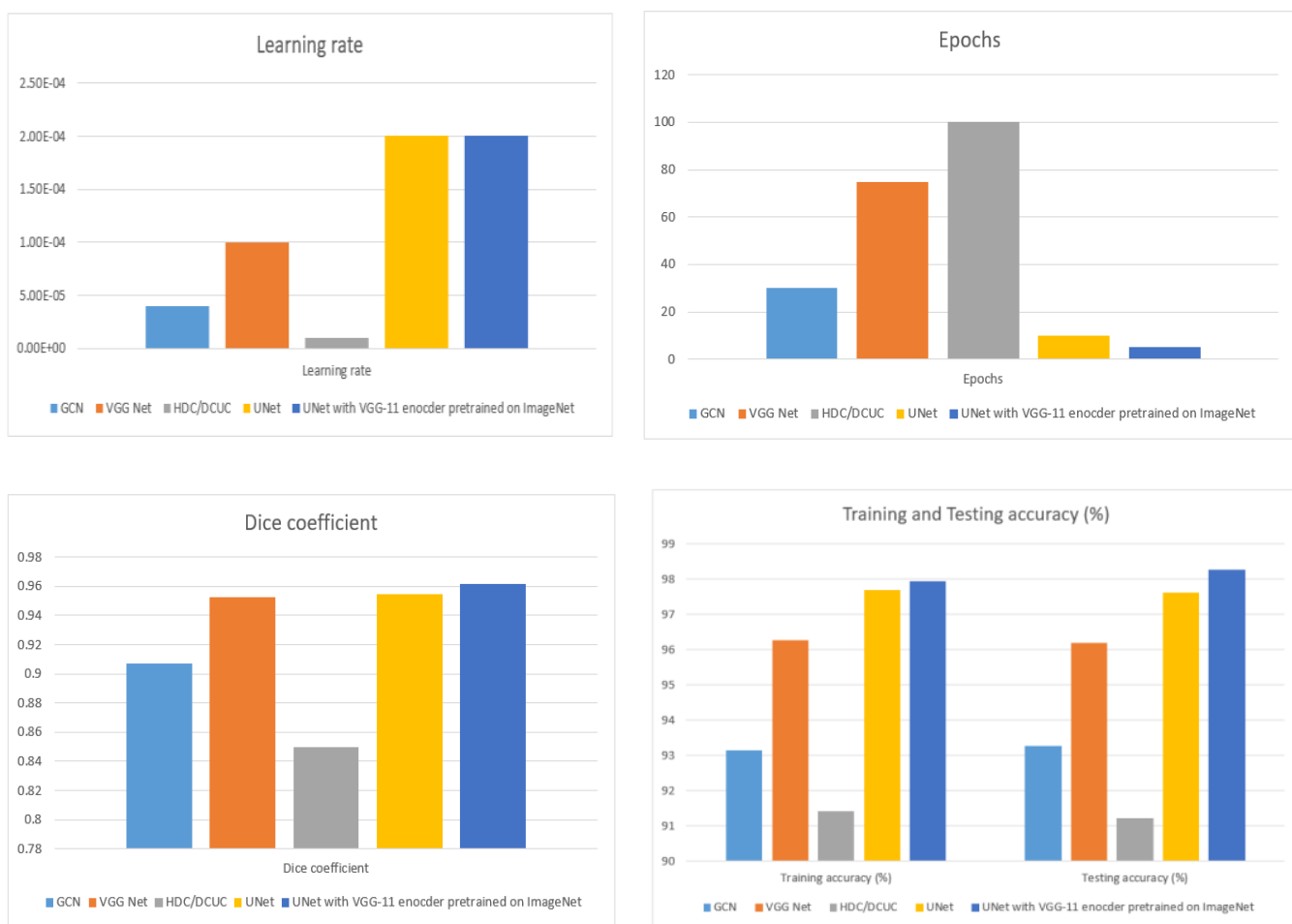

Fig.16. The graph compared for existing and proposed system

\section{Conclusion}

Convolutional neural network methods are attempted for segmentation of the lungs in this work. Several performance metrics such as dice coefficient, Validation and Training Accuracy were utilized in order to determine the models performance. Also compared the UNet architecture and the UNet with VGG-11 encoder pre-trained 
with ImageNet with the other architectures such as GCN, VGG Net and HDC/DCUC. The higher accuracy obtained is $97.63 \%$ for UNet and $98.28 \%$ for the UNet with VGG-11 encoder pre-trained with ImageNet respectively.

\section{Declarations \\ Source of Funding sectors. \\ Competing Interests Statement \\ Consent to participate \\ Not Applicable \\ Consent for publication}

This research did not receive any specific grant from funding agencies in the public, commercial, or not-for-profit

The authors declare no competing financial, professional and personal interests.

We declare that we consented for the publication of this research work.

\section{Availability of data and material}

Authors are willing to share data and material according to the relevant needs.

\section{References}

[1] Hieu Trung Huynh; VoNguyen Nhat Anh; A Deep Learning Method for Lung Segmentation on Large Size Chest X-Ray Image, IEEE-RIVF International Conference on Computing and Communication Technologies (RIVF), 10.1109/RIVF.2019.8713648, 2019.

[2] Jonnison Lima Ferreira; Giovanni Lucca França da Silva; An automatic method for lung segmentation and reconstruction in chest X-ray using deep neural networks, Computer Methods and Programs in Biomedicine, 2019.

[3] Mingjie Xu,Shouliang Qi LinMing-Ching Lee; Christoph Palm; Semantic Lung Segmentation Using Convolutional Neural Networks, Bildverarbeitung für die Medizin 2020, 10.1007/ 978-3-658-29267-6_17, 2020.

[4] R. D. S. Portela; J. R. G. Pereira; M. G. F. Costa; C. F. F. Costa Filho, Lung Region Segmentation in Chest X-Ray Images using Deep Convolutional Neural Networks, 42nd Annual International Conference of the IEEE Engineering in Medicine \& Biology Society (EMBC), 10.1109/EMBC44109.2020.9175478, 2020.

[5] Ching-Sheng Chang, Jin-Fa Lin,Ming - Ching Lee,Christoph Palm ,2020(Springer Paper).

[6] Nihad Mesanovic; Svjetlana Mujegic; Application of lung segmentation algorithm to disease quantification from CT images, International Conference on System Engineering and Technology (ICSET), 10.1109/ICSEngT.2012.6339340, 2012.

[7] Yambem Jina Chanu Y. Le Cun, Y. Bengio and G. Hinton, Deep learning, Nature 521, 436444, 2015. 
[8] B. Bischke, P. Helber, J. Folz, D. Borth and A. Dengel, Multi-Task Learning for Segmentation of Building Footprints with Deep Neural Networks, arXiv:1709.05932, 2017.

[9] K. Simonyan and A. Zisserman, Very Deep Convolutional Networks for Large-Scale Image Recognition, arXiv:1409.1556, 2014.

[10] Maggiori, Y. Tarabalka, G. Charpiat and P. Alliez, Can Semantic Labeling Methods Generalize to Any City? The Inria Aerial Image Labeling Benchmark, hal.inria.fr/hal-01468452, IGARSS, 2017.

[11] Ching-Sheng Chang, Jin-Fa Lin,Ming ,K. Nakagami, A. Shimizu, H. Kobatake, M. Yakaomi, K. Fujimoto, and $\mathrm{K}$. Togashi, "Multi-shape graph cuts with neighbor prior constraints and its application to lung segmentation from a chest CT volume," Med. Image Anal., vol. 17, no. 1, pp. 62-77, Jan. 2013.

[12] O.Ronneberger, P. Fischer, and T. Brox, "U-Net: Convolutional Networks for Biomedical Image Segmentation,” Med. Image Comput. Comput. Interv. -- MICCAI 2015, pp. 234-241, 2015.

[13] D. Kingma and J. Ba, Adam: A Method for Stochastic Optimization, arXiv:1412.6980, 2014.

[14] Z. Akkus, A. Galimzianova, A. Hoogi, D. L. Rubin, and B. J. Erickson, "Deep Learning for Brain MRI Segmentation: State of the Art and Future Directions," Journal of Digital Imaging, vol.30, no.4. pp. 449-459, 2017. [15] M. Havaei et al., "Brain tumor segmentation with Deep Neural Networks," Med. Image Anal., vol. 35, pp. 18 $31,2017$.

[16] K. He, X. Zhang, Sh. Ren and J. Sun, Deep Residual Learning for Image Recognition, arXiv:1512.03385, 2015. 